\title{
High short-term mortality following lung biopsy for usual interstitial pneumonia
}

\author{
J.P. Utz*, J.H. Ryu*, W.W. Douglas*, T.E. Hartman", H.D. Tazelaar", J.L. Myers", \\ M.S. Allen ${ }^{+}$, D.R. Schroeder ${ }^{\S}$
}

\begin{abstract}
High short-term mortality following lung biopsy for usual interstitial pneumonia. J.P. Utz, J.H. Ryu, W.W. Douglas, T.E. Hartman, H.D. Tazelaar, J.L. Myers, M.S. Allen, D.R. Schroeder. C ERS Journals Ltd 2001.

ABSTRACT: Usual interstitial pneumonia (UIP) is a specific histological pattern of interstitial pneumonia most often associated with the clinical syndrome of idiopathic pulmonary fibrosis (IPF). There is controversy regarding the use of surgical lung biopsy in the diagnosis of UIP, and the risk of lung biopsy in these patients is largely unknown. This study investigated the $\mathbf{3 0}$ day surgical mortality rate in patients undergoing surgical lung biopsy for UIP.

Patients undergoing surgical lung biopsy over a 10-yr period from 1986-1995 with the ultimate diagnosis of UIP (with or without underlying connective tissue disease) were identified. Pathology, computed tomography, medical records, and survival were assessed.

Ten of sixty patients with usual interstitial pneumonia were found to be dead within 30 days of surgical biopsy. All of these were patients with idiopathic UIP, unassociated with connective tissue disease (clinical condition of IPF).

In conclusion, patients with usual interstitial pneumonia of the idiopathic type, who present with atypical features, may be at higher risk for death following surgical biopsy than patients presenting with more typical features or patients with other interstitial illnesses.
\end{abstract}

Eur Respir J 2001; 17: 175-179.

\begin{abstract}
*Division of Pulmonary and Critical Care Mediçine, "Dept of Diagnostic Radiology, ${ }^{\top}$ Dept of Pathology, ${ }^{+}$Division of General Thoracic Surgery and ${ }^{\S}$ Division of Biostatistics, Mayo Clinic Rochester, Minnesota, USA.
\end{abstract}

Correspondence: J.P. Utz

Division of Pulmonary and Critical Care Medicine

Mayo Clinic

Rochester

Minnesota

USA

Fax: 15072664372

Keywords: Idiopathic pulmonary fibrosis

mortality

usual interstitial pneumonia

Received: November 221999

Accepted after revision September 15 2000
Usual interstitial pneumonia (UIP) is a specific pattern of interstitial pneumonia that typically occurs in patients with the clinical syndrome of idiopathic pulmonary fibrosis (IPF) [1 -4]. UIP can also occur in association with underlying connective tissue diseases, occupational asbestos exposure (i.e. asbestosis), and with certain drug toxicities [2]. Historically, UIP was considered to be the underlying histology in a subset of patients with IPF; however, current recommendations are that the diagnosis of IPF be restricted to those patients with unexplained UIP (UIP/IPF). The prognosis for patients with UIP/IPF is poor, with a median survival of $\sim 2.8$ yrs [5].

In many studies, the diagnosis of UIP has been based upon a combination of characteristic clinical and radiographic features, with histologic confirmation in only selected cases [2,6-10]. Others have advocated wedge biopsies in all patients suspected of having UIP/ IPF, especially in those patients $<60 \mathrm{yrs}$ of age [1]. High resolution computed tomography (HRCT) of the chest has reduced the proportion of patients undergoing biopsy procedures, such that many patients with a compatible clinical history and physical examination, and an HRCT typical of UIP, are often not subjected to surgical lung biopsy [11]. The relative accuracy of HRCT versus surgical biopsy in the diagnosis of UIP is controversial [2, 12-21]. The Mayo Clinic has generally accepted a clinicoradiographic diagnosis of UIP/IPF in patients with typical clinical features and a "classic" appearance on HRCT. The risk of surgical lung biopsy in patients with UIP is largely unknown. This study, therefore, reviewed the 30 day surgical mortality rate in all patients with UIP at the Mayo Clinic, on lung biopsies obtained by either thoracotomy or videoassisted thoracoscopic surgery (VATS) during a 10-yr period (1986-1995).

\section{Methods}

\section{Patient selection}

All pathology reports for lung tissue removed by open thoracotomy or VATS at the Mayo Clinic, Rochester were reviewed for the years 1986-1995. Of 3,736 reports, 68 included the diagnosis of UIP. These 68 specimens were jointly reviewed by two of the authors (H.D. Tazelaar and J.L. Myers), without knowledge of the clinical or radiological findings. Only patients with histologically confirmed UIP were retained in the study population. 


\section{Pathology review}

Criteria for the diagnosis of UIP included a heterogeneous appearance at low magnification resulting from alternating zones of normal lung, patchy interstitial widening due to a combination of inflammation and fibrosis, foci of recent organization with fibroblast proliferation ("fibroblast foci"), and honeycomb changes $[2,3,5]$. The fibrotic changes preferentially affected subpleural and paraseptal parenchyma.

\section{Medical record review}

All medical records related to an individual patient at the Mayo Clinic are contained in a single unit record. These records contain all inpatient and outpatient records, laboratory results and correspondence. These records were reviewed and relevant information recorded including age at biopsy, sex, type of biopsy, vital status at last follow-up, preoperative pulmonary function data, clinical-pathological diagnosis, and radiographic imaging dates and results. The reason for biopsy was determined and recorded, as best as it could be in a retrospective fashion. A mailed survey, supplemented by telephone interview of patients or relatives, was utilized to obtain vital status information for all patients not known to be deceased at the time of last contact. Date of death was obtained from death certificates or next of kin. Vital status was determined on all patients reviewed, no patient was lost to followup.

\section{Radiographic review}

All available Mayo and outside computed tomographic (CT) scans of the chest were reviewed jointly by two readers (T.E. Hartman and J.P. Utz) in the absence of clinical information. Patients in whom HRCT images were available for review were classified as (1) diagnostic for UIP, (2) possibly UIP, or (3) not consistent with UIP. HRCT criteria for a confident diagnosis of UIP included symmetric, lower lung predominant, bilateral irregular linear opacities occurring in a subpleural distribution with associated honeycombing. Traction bronchiectasis and areas of ground glass attenuation could be present, but were not required for diagnosis. CT studies were classified as "possible UIP" if the irregular linear opacities lacked a lower lung predominance or subpleural distribution, or if the irregular linear opacities were asymmetric or if honeycombing was not present. CT scans interpreted as "not consistent with UIP" demonstrated findings other than those noted above.

\section{Statistical analysis}

Cumulative survival probabilities were estimated using the Kaplan-Meier method [22]. Patients that died within 30 days following biopsy were compared to those that survived beyond 30 days, using the rank sum test for continuous variables and Fisher's exact test for categorical variables. In all cases, two-tailed p-values $\leqslant 0.05$ were considered statistically significant.

\section{Results}

Pathological review confirmed the diagnosis of UIP in 60 of 68 cases. Eight patients were excluded from further analysis because their biopsies showed nonspecific interstitial pneumonia (4 patients), sarcoidosis (1), nonspecific scarring with adenocarcinoma (1), nonspecific scarring with emphysema (1), and a nonclassifiable interstitial pneumonia (1). Among the 60 patients with UIP, there were 41 males and 19 females. The age at time of biopsy was $63.4 \pm 10.5 \mathrm{yrs}$ $($ mean $\pm \mathrm{SD})$. Forty-four $(73 \%)$ biopsies were obtained by open thoracotomy, and $16(27 \%)$ were obtained by VATS. Baseline pulmonary function testing at time of biopsy is shown in table 1. Clinicopathological diagnoses included UIP/IPF in 46 patients $(76.7 \%)$ and UIP associated with connective tissue disease (UIP/CTD) in 14 patients $(23.3 \%)$. The associated connective tissue diseases included primary systemic sclerosis (4 patients), dermatomyositis/polymyositis (4), rheumatoid arthritis (2), mixed connective tissue disease (1), suspected connective tissue disease with nonspecific features (2), and uncharacterized vasculitis (1). Five of the 60 patients had evidence of diffuse alveolar damage (DAD) superimposed on underlying UIP. Of these 5 patients, 4 had UIP/IPF and 1 had UIP/CTD.

The majority of patients were biopsied because of atypical clinical or radiographic features, or because of "diagnostic uncertainty". Seven patients were primarily biopsied in order to rule out a secondary process (i.e. infection) based upon an accelerated decline in function or a change in the character of radiographic infiltrates. Aside from DAD, no secondary process was identified in any patient. Some patients were biopsied to obtain a "definitive diagnosis" or to "define activity" of disease, generally prior to considering a prolonged course of corticosteroids. Five patients had lung biopsies coincident with other thoracic surgery. These indications included cardiac surgery in two patients (mitral valve replacement and mitral valve replacement with coronary artery bypass grafting), lung cancer surgery, pleurodesis for pneumothorax, and take-down of a failed Nissen fundoplication, in a single patient each.

Twenty-eight patients $(46.7 \%)$ had a preoperative HRCT, eight had only a conventional CT scan (13.3\%), and $24(40.0 \%)$ had no preoperative CT scan. Cases in which only conventional CT images were available were intrinsically unable to achieve a label of "consistent with UIP" since these criteria are based on HRCT features. Of the 28 patients with HRCT scans prior to lung biopsy, 25 were available for review. Three $(12 \%)$ were considered diagnostic of UIP, $17(68 \%)$ were possibly UIP, and $5(20 \%)$ were not consistent with UIP.

Ten of 60 patients with UIP were dead within 30 days of biopsy $(16.7 \%, 95 \%$ exact confidence interval $8.3-28.5 \%$ ). These included three of 16 patients subjected to VATS biopsy $(18.8 \%)$ and seven of 44 $(15.9 \%)$ patients subjected to thoracotomy and biopsy. All of the patients that died within 30 days following biopsy were in the UIP/IPF group $(10 / 46,21.7 \%$, versus 
Table 1.-Baseline pulmonary function at biopsy

\begin{tabular}{lcccc}
\hline & $\begin{array}{c}\text { All patients } \\
(\mathrm{n}=60)\end{array}$ & $\begin{array}{c}\text { Alive after 30 days } \\
(\mathrm{n}=50)\end{array}$ & $\begin{array}{c}\text { Dead after 30 days } \\
(\mathrm{n}=10)\end{array}$ & $\mathrm{p}$-value \\
\hline Age yrs & $63.4 \pm 10.5$ & $63.7 \pm 10.8$ & $62.0 \pm 8.9$ & 0.592 \\
FVC L & $2.53 \pm 0.92(56)$ & $2.53 \pm 0.92(48)$ & $2.57 \pm 0.95(8)$ & 0.752 \\
FVC \% predicted of normal & $66.5 \pm 18.0(55)$ & $67.1 \pm 17.5(47)$ & $63.3 \pm 21.9(8)$ & 0.702 \\
DLCO mmol-min ${ }^{-1} \cdot \mathrm{kPa}^{-1}$ & $1.68 \pm 0.62(53)$ & $1.73 \pm 0.62(46)$ & $1.31 \pm 0.53(7)$ & 0.107 \\
DLCO \% predicted of normal & $49.5 \pm 18.9(40)$ & $52.1 \pm 18.4(34)$ & $34.7 \pm 14.8(6)$ & 0.028 \\
\hline
\end{tabular}

Data are presented as means \pm SD (number of patients with available data); ${ }^{\#}$ : Rank sum test.

$0 / 10$ for the UIP/CTD group, $\mathrm{p}=0.098$ ). Four of the five patients with DAD on UIP were in the IPF group, and two of the four died within 30 days, while the remaining two died within four months of biopsy. The fifth patient with DAD on UIP had primary systemic sclerosis (PSS) and was alive at 87 months after biopsy. A lower diffusing capacity for carbon monoxide (DLCO) was predictive of death within 30 days with a mean DLCO of $52.1 \%$ predicted in the 30 days survivors versus $34.7 \%$ predicted in the patients dead within 30 days $(p=0.028)$. Cause of death in all 10 patients dead within 30 days of biopsy was attributed to a respiratory cause, most often adult respiratory distress syndrome (table 2).

Four of 10 patients dead within 30 days of biopsy had a clinical diagnosis of IPF prior to biopsy and were biopsied because of an accelerated decline in respiratory status. One of these four was found to have DAD on UIP, while the remaining three were found to have UIP only. Two additional patients were biopsied in the setting of a rapidly worsening course of dyspnoea and infiltrates, but preoperatively, there was diagnostic uncertainty regarding the underlying process and they were therefore not considered to have a worsening UIP/ IPF. One of these showed UIP on biopsy while the other was found to have DAD and UIP. Another patient with known UIP (open lung biopsy 4 yrs earlier) with subacute worsening was subjected to biopsy during coronary artery bypass grafting (CABG) and mitral valve repair for a flail leaflet, making it difficult to determine whether the decline was secondary to a cardiac or respiratory cause.

\section{Discussion}

Most patients seen at the Mayo Clinic with a suspected diagnosis of UIP are not subjected to lung biopsy by thoracotomy or VATS. For this analysis, patients with bronchoscopic lung biopsies only were excluded, because these small biopsies are not accurate for diagnosing UIP [23]. This study's population comprises a small fraction of all patients with UIP seen at our institution during this 10 -yr period. The precise fraction of UIP patients biopsied during this period (1986-1995) is not known, but is thought to be a distinct minority. These patients were subjected to biopsy, largely because they did not demonstrate typical, historical or physical examination findings. Some were biopsied because of clinical decline in an attempt to rule out secondary processes. Others had atypical CT features, most often, a lack of honeycomb change.

Table 2. - Patients dead within 30 days

\begin{tabular}{|c|c|c|c|c|}
\hline Age/sex & $\begin{array}{l}\text { Preoperative } \\
\text { diagnosis }\end{array}$ & Reason for biopsy & Biopsy result & Cause of death \\
\hline $61 / \mathrm{M}$ & IPF & AD R/O secondary mass & UIP & $\begin{array}{l}\text { Increase in infiltrate, bleeding, } \\
\text { unresponsive one day after TOCP }\end{array}$ \\
\hline $71 / \mathrm{M}$ & IPF & AD R/O secondary process & DAD on UIP & $\begin{array}{l}\text { Respiratory failure, } \\
\text { never extubated* }\end{array}$ \\
\hline 69/M & IPF & AD R/O secondary process & UIP & $\begin{array}{l}\text { Respiratory failure, } \\
\text { probable ARDS* }\end{array}$ \\
\hline $68 / \mathrm{M}$ & IPF & AD R/O secondary process & UIP & ARDS \\
\hline $67 / \mathrm{F}$ & BOOP/DAD? ${ }^{\#}$ & DU Subacute course & DAD on UIP & ARDS \\
\hline 64/M & UIP & $\begin{array}{l}\text { AD (but myocardial infarction, } \\
\text { flail mitral leaflet) }\end{array}$ & UIP & Respiratory failure \\
\hline $64 / \mathrm{F}$ & AIP? & $\begin{array}{l}\text { DU Subacute course, alveolar } \\
\text { infiltrates }\end{array}$ & $\mathrm{UIP}^{+}$ & ARDS \\
\hline $58 / \mathrm{M}$ & $\begin{array}{l}\text { UIP/eosinophilic } \\
\text { granuloma? }\end{array}$ & DU Atypical CT distribution & $\mathrm{UIP}^{+}$ & $\begin{array}{c}\text { Acute pulmonary oedema } \\
\text { or severe UIP }\end{array}$ \\
\hline 40/M & IPF? & DU Atypical y & $\mathrm{UIP}^{+}$ & ARDS \\
\hline $58 / \mathrm{M}$ & $\begin{array}{l}\text { IPF/eosinophilic } \\
\text { granuloma/Sarcoidosis }\end{array}$ & $\begin{array}{c}\text { DU Upper lobe predominant } \\
\text { infiltrates }\end{array}$ & UIP & $\begin{array}{l}\text { Pneumothorax/ } \\
\text { Empyema/sudden death }\end{array}$ \\
\hline
\end{tabular}

*: autopsy declined; ${ }^{*}$ : bronchiolitis obliterans organizing pneumonia/diffuse alveolar damage; ": open lung biopsy 4 years prior; ${ }^{+}$: indicates video assisted thoracoscopic biopsy, all others open surgical biopsy; IPF: idiopathic pulmonary fibrosis; UIP: usual interstitial pneumonia; DAD: diffuse alveolar damage; ARDS: adult respiratory distress syndrome; BOOP: bronchiolitis obliterans organizing pneumonia; AIP: acute interstitial pneumonia; AD: accelerated decline; DU: dignostic uncertainty; TOCP: transtracheal oxygen catheter placement; ?: uncertain diagnosis. 
The fact that $40 \%$ of biopsied patients did not have preoperative CT may reflect the atypical presentation of these patients, the perceived urgency to biopsy, or the time frame studied (1986 - 1995). A more recent analysis of 487 patients with UIP seen at our institution from $1994-1996$ indicated that $94.7 \%$ of patients underwent CT imaging [24]. Therefore, the patients who underwent thoracotomy or VATS for UIP during this time period represent a highly selected group that may not reflect the greater population of patients with underlying UIP. A more recent review of patients with UIP at our institution during the years 1994-1996 indicated that approximately $20 \%$ of patients with a diagnosis of UIP had been subjected to surgical biopsy [24].

The overall 30-day mortality rate for this group of patients is surprisingly high, given the low historical surgical mortality rates previously reported from the Mayo Clinic. In an analysis of 771 patients undergoing VATS at the Mayo Clinic from 1991-1994, Allen et al. [25] observed a $1.9 \%$ overall 30 -day mortality rate, which compares favourably with other reported surgical mortality rates for VATS [26, 27]. Interestingly, all deaths reported by ALLEN et al. [25] in patients undergoing VATS biopsy occurred in those with diffuse lung infiltrates, though all of these patients had preoperative respiratory failure. In that study, of the 234 patients who underwent VATS wedge resection for a pulmonary nodule (no conversion to an open procedure), there were no surgical deaths. All deaths $(\mathrm{n}=15)$ occurred in patients undergoing VATS wedge biopsy for diffuse pulmonary infiltrates (types not specified). Wedge specimens (147) were collected from patients with diffuse infiltrates, though some patients may have had more than one specimen (the precise number of patients with diffuse infiltrates was not specified). Therefore, the mortality rate in these patients with diffuse infiltrates was at least $10 \%(15 / 147)$, if one assumes only one wedge specimen per patient. If any patients had more than one specimen, the number of patients represented by 147 specimens drops, thereby increasing the calculated mortality. Recent 30-day surgical mortality rates at the Mayo Clinic were $0.4 \%$ after lobectomy, $2.7 \%$ following wedge resection, and $5.4 \%$ after pneumonectomy (personal communication, M.S. Allen, Division of General Thoracic Surgery, Mayo Clinic, Rochester, MN, USA).

All short-term mortality in this study occurred in patients with UIP/IPF rather than UIP/CTD. Indeed, the 30-day mortality rate for patients with UIP/IPF was $21.7 \%$ (10/46). Explanations for the high surgical mortality rate in this population of patients are largely speculative. There did not appear to be any differences in surgical technique, anaesthesia, or postoperative care between patients who died within 30 days of biopsy and the survivors. The authors are unaware of any variance in the thoracotomy or VATS technique in this group of patients versus others subjected to thoracotomy or VATS procedures with other conditions. It is possible that the high 30-day surgical mortality rate reflects more severe lung disease in patients undergoing a surgical biopsy compared to those not subjected to biopsy. Lower DL,CO (\% predicted) was the only preoperative parameter that showed a significant correlation with the risk of postoperative death in biopsied patients $(\mathrm{p}=0.028)$. Other pulmonary function parameters were not statistically different. Seven patients were biopsied because of accelerated disease progression, suggesting that this subset of patients may be at higher risk of early postoperative death. The presence of DAD and UIP in open lung biopsies has been described in patients with accelerated deterioration or acute exacerbation of IPF [28, 29]. In the present study this combination of findings did not necessarily correlate with either a clinical history of accelerated disease, or early postoperative death, although all but one had died within 4 months of biopsy.

Although it is difficult to be certain, given the selection bias inherent in a retrospective analysis of this sort, the present findings suggest that patients with UIP presenting with atypical features may be at higher risk for death following open thoracotomy or VATS biopsy than those presenting with typical features or patients with other illnesses. Two other reports offer additional evidence that the risk of surgical biopsy in these patients may be higher than currently appreciated. SALAZARFlores and Salas-Hernandez [30] reported in abstract form the causes of death in 110 patients with "pulmonary fibrosis". While details of this series are not known, $13.6 \%$ of deaths in these 110 patients were attributed to complications of biopsy. TAZELAAR et al. [31] described three histologic subtypes of patients with polymyositis and dermatomyositis, including broncholitis obliterans-organizing pneumonia, DAD, and UIP. Of the patients with UIP on surgical biopsy, $60 \%(3 / 5)$ were dead within 30 days of biopsy, indicating that patients with UIP/CTD may also be at increased risk, despite the absence of early postoperative deaths in the present study's reported patients with UIP/CTD.

It is difficult to formulate specific recommendations in the absence of large prospective studies evaluating the risk of lung biopsy in patients with suspected usual interstitial pneumonia. The authors would urge others to report their own experience with wedge lung biopsy in their own patients to further discern whether patients with usual interstitial pneumonia may be subject to a previously unappreciated high risk following surgical biopsy. This may be particularly crucial since, in current practice, surgical biopsy is often done in a minority of patients and recommendations suggest surgical biopsy in patients with presentations atypical for usual interstitial pneumonia [8, 24, 32-34]. The present findings indicate that this subset of patients may be at higher risk for surgical biopsy than previously recognized.

\section{References}

1. Reynolds HY. Diagnostic and management strategies of diffuse interstitial lung disease. Chest 1998; 113: $192-202$.

2. Ryu JH, Colby TV, Hartman TE. Idiopathic pulmonary fibrosis: current concepts. Mayo Clin Proc 1998; 73: 1085 - 1101.

3. Katzenstein AA, Myers JL. Idiopathic pulmonary fibrosis: clinical relevance of pathologic classification. Am J Respir Crit Care Med 1998; 157: 1301-1315.

4. Katzenstein AA. Idiopathic interstitial pneumonia: 
classification and diagnosis. Monog in Pathol 1993; 36 : $1-31$.

5. Bjoraker JA, Ryu JH, Edwin MK, et al. Prognostic significance of histopathologic subsets in idiopathic pulmonary fibrosis. Am J Respir Crit Care Med 1998; 157: $199-203$.

6. Mathieson JR, Mayo JR, Staples CA, Müller NL. Chronic diffuse infiltrative lung disease. Comparison of diagnostic accuracy of CT and chest radiography. Radiology 1989; 171: 111-116.

7. Hansell DM, Wells AU. CT evaluation of fibrosing alveolitis. Application and insights. $J$ Thorac Imag 1996; 11: $231-249$.

8. Johnston IDA, Gomm SA, Kalra S, Woodcock AA, Evans CC, Hind CRK. The management of cryptogenic fibrosing alveolitis in three regions of the United Kingdom. Eur Respir J 1993; 6: 891-893.

9. Akira M, Sakatani M, Ueda E. Idiopathic pulmonary fibrosis. Progression of honeycombing at thin-section CT. Radiology 1993; 189: 687-691.

10. Johnston IDA, Prescott RJ, Chalmers JC, Rudd RM. British Thoracic Society study of cryptogenic fibrosing alveolitis. Current presentation and initial management. Thorax 1997; 52: $38-44$.

11. Chan-Yeung M, Muller NL. Cryptogenic fibrosing alveolitis. Lancet 1997; 350: 651-656.

12. Cherniack RM, Colby TV, Flint A, et al. Quantitative assessment of lung pathology in idiopathic pulmonary fibrosis. Am Rev Respir Dis 1991; 144: 892-900.

13. Nishimura K, Kitaichi M, Izumi T, Nagai S, Kanaoka $\mathrm{M}$, Itoh H. Usual interstitial pneumonia. Histologic correlation with high-resolution CT. Radiology 1992; 182: $337-342$.

14. Nishimura K, Izumi T, Kitaichi M, Nagai S, Itoh $H$. The diagnostic accuracy of high-resolution computed tomography in diffuse infiltrative lung diseases. Chest 1993; 104: 1149-1155.

15. Lynch DA, Newell JD, Logan PM, King TE, Müller NL. Can CT distinguish hypersensitivity pneumonitis from idiopathic pulmonary fibrosis? AJR 1995; 165 : $807-811$.

16. Miller RR, Webb WR, Evans KG, Ostrow DN. Fibrosing alveolitis: CT-pathologic correlation. Radiology 1986; 160: 585-588.

17. Strickland B, Strickland NH. The value of high definition, narrow section computed tomography in fibrosing alveolitis. Clin Radiol 1988; 39: 589-594.

18. Tung KT, Wells AU, Rubens MB, Kirk JME, du Bois RM, Hansell DM. Accuracy of the typical computed tomographic appearances of fibrosing alveolitis. Thorax 1993; 48: 334-338.

19. Colby TV, Swensen SJ. Anatomic distribution of histopathologic patterns in diffuse lung disease: correlation with HRCT. $J$ Thorac Imag 1996; 11: $1-$ 26.

20. Swensen SJ, Aughenbaugh GL, Myers JL. Diffuse lung disease: diagnostic accuracy of CT in patients undergoing surgical biopsy of the lung. Radiology 1997; 205: 229-234.

21. Orens JB, Kazerooni EA, Martinez FJ, et al. The sensitivity of high-resolution CT in detecting idiopathic pulmonary fibrosis proved by open lung biopsy: a prospective study. Chest 1995; 108: $109-115$.

22. Kaplan E, Meier P. Nonparametric estimation from incomplete observations. JASA 1958; 53: 457-481.

23. Wall CP, Gaensler EA, Carrington CB, Hayes JA. Comparison of transbronchial and open biopsies in chronic infiltrative lung diseases. Am Rev Respir Dis 1981; 123: 280-285.

24. Douglas WW, Ryu JH, Schroeder DR. Idiopathic pulmonary fibrosis: Impact of oxygen and colchicine, prednisone or no therapy on survival. Am J Respir Crit Care Med 2000; 161: 1172-1178.

25. Allen MS, Deschamps C, Jones DM, Trastek VF, Pairolero PC. Video-assisted thoracic surgical procedures: the Mayo experience. Mayo Clin Proc 1996; 71: $351-359$.

26. Harris RJ, Kavuru MS, Rice TW, Kirby TJ. The diagnostic and therapeutic utility of thoracoscopy. A review. Chest 1996; 108: 828-841.

27. Hazelrigg SR, Nunchuck SK, LoCicero J. Video assisted thoracic surgery study group data. Ann Thorac Surg 1993; 56: 1039-1044.

28. Akira M, Hamada H, Sakatani M, Kobayashi C, Nishioka M, Yamamoto S. CT findings during phase of accelerated deterioration in patients with idiopathic pulmonary fibrosis. AJR 1997; 168: 79-83.

29. Kondoh Y, Taniguchi H, Kawabata Y, Yokoi T, Suzuki K, Takagi K. Acute exacerbation in idiopathic pulmonary fibrosis. Analysis of clinical and pathologic findings in three cases. Chest 1993; 103: $1808-1812$.

30. Salazar-Flores M, Salas-Hernandez J. Causes of death in 110 patients with pulmonary fibrosis. Am J Respir Crit Care Med 1998; 157: A279.

31. Tazelaar HD, Viggiano RW, Pickersgill J, Colby TV. Interstitial lung disease in polymyositis and dermatomyositis: clinical features and prognosis as correlated with histologic findings. Am Rev Respir Dis 1990; 141: 727-733.

32. American Thoracic Society. Idiopathic pulmonary fibrosis: Diagnosis and treatment (International consensus statement). Am J Respir Crit Care Med 2000; 161: 646-664.

33. Raghu G, Mageto YN, Lockhart D, Schmidt RA, Wood DE, Godwin JD. The accuracy of the clinical diagnosis of new-onset idiopathic pulmonary fibrosis and other interstitial lung disease: A prospective study. Chest 1999; 116: $1168-1174$.

34. British Thoracic Society recommendations. The diagnosis, assessment and treatment of diffuse parenchymal lung disease in adults. Thorax 1999; 54: $\mathrm{S} 1-\mathrm{S} 30$. 